\title{
Climate Change Impacts on the Common Swift in South Africa
}

\author{
Danni Guo, Gina Zietsman, and Philip A. R. Hockey
}

\begin{abstract}
The Common Swift Apus apus, a species that has undergone major range expansion within South Africa in the past few decades, provides an opportunity to determine whether this change in range was indeed driven by changes in climate. This question was explored using species distribution modelling, combining predictive and retrodictive approaches. Using distribution and climate in the 1960s as the baseline, climate models were used to predict range changes of the swifts at decadal intervals into the 2000 s, which were then compared with observed ranges of the swifts at the same time intervals. Based on comparisons between patterns of changing climate and changes in both model predicted distributions and observed distribution patterns, the species distribution model proves to have sufficient power to predict the species' range expansion. This study thus provides compelling evidence for a major range change of a bird species in South Africa being driven primarily by climatic factors.
\end{abstract}

Index Terms-Climate change, common swift, range shift, South Africa.

\section{Climate Change And the Common SwiFT}

Climate change has been more accepted as a real phenomenon in the recent years, and as an important influence over plant and animal species distribution and range shifts. Due to climate change, the ranges of many bird species are changing, and predicting the patterns of these changes is important in developing proactive conservation strategies. While species distribution models has been readily available, and has proven to be accurate and useful in modelling species distributions, given a good sample data set. However, field scientists do question its accuracy and predictive abilities when it comes to species predictions for the future. This study is important to confirm our observation that most species' range shift can be predicted if long term study were done on tracking the climate change impact on the species.

The Common Swift (European Swift) Apus apus is a medium sized Swift, 16-18 cm with long, scythe-like wings and forked tail, and it is black-brown except for a small white or grey patch on the chin (Fig. 1). Its geographic range

Manuscript received April 12, 2015; revised June 18, 2015. This work was supported in part by the National Research Foundation (NRF) Funding Reference: IFR150206113775 Grant number: 96163.

Danni Guo is with Biodiversity Research Assessment and Monitoring, South African National Biodiversity Institute, Kirstenbosch Research Center, Private Bag X7, Claremont 7735, South Africa (e-mail: d.guo@sanbi.org.za).

Gina Zietsman was with the Percy FitzPatrick Institute, DST/NRF Centre of Excellence, University of Cape Town, Rondebosch 7701, South Africa (e-mail: gina@mossgroup.co.za).

Philip A. R. Hockey was with the Percy FitzPatrick Institute, DST/NRF Centre of Excellence, University of Cape Town, Rondebosch 7701, South Africa. spreads over Europe, Asia, and Africa. It is a migratory species, and it breeds at northern latitudes and travels to Southern Africa before the onset of the northern winter, and the non-breeding grounds range over whole of Southern Africa. It is entirely aerial during their residency period in South Africa feeding on insects and airborne spiders [1]. Because the species is a non-breeding visitor to the region, its distribution is unconstrained by needing access to breeding sites. It is also an exclusively aerial forager, which drinks and sleeps on the wing: it is therefore unconstrained by a need for terrestrial roost sites, and therefore they are effectively independent of habitat structure. The swifts are known to be sensitive to temperature changes, and not so much by precipitation.

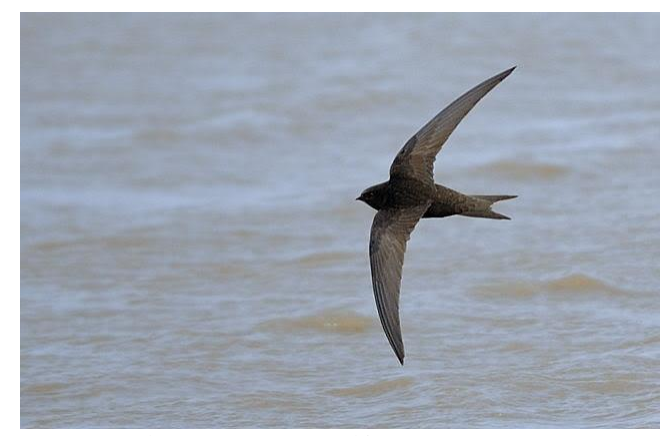

Fig. 1. Common swift Apus apus [1].

The southern regions of South Africa have seen significant warming trends over the past 50 years, but despite these changes in Africa's climate, the impacts on African birds have been little explored. In southern Africa, one of the most extensive range changes has been that of the common swift, a non-breeding summer migrant to the region whose range has extended south-westwards since the 1960s, colonising primarily the semi-arid western regions of southern Africa While range shifts can and do occur for reasons other than climate change, but the case of common swifts, however, there is no evidence for global population size increase [2].

The primary aim of the study was to generate parallel reconstructions over time of the changing range of common swift and the changing climate of South Africa, and to assess whether a species distribution modelling approach could have predicted the future patterns of range change. The study will provide the first empirical test of whether a South African bird's range has been influenced by climate change.

\section{TEMPERATURE RECONSTRUCTION IN SOUTH AFRICA}

Temperature was chosen as the primary explanatory variable because it imposes constraints on swift's distributions [3]. Since we have already established that the swifts are 
sensitive to temperature changes and not so much by precipitation, we need to create the decadal temperature layers needed for species distribution modelling. Besides the temperature variable, altitude was added as a second constraint.

The temperature data used in this paper was provided by the Climate Systems Analysis Group (CSAG) based at the University of Cape Town and the South African Weather Service (SAWS). The weather stations were selected based on their even spread as over the country and on the availability of the data. The weather stations were used on the condition that the temperature data were available over at least a decade, and the mean monthly maximum and minimum temperatures were recorded. For each station, monthly temperatures over the four months of the swift's main residency period, December to March, were calculated by averaging the mean monthly maximum and minimum temperatures to produce an average summer temperature for each year. These seasonal averages were then converted to decadal summer temperature means for each station, and these decadal temperature averages were used in estimating swift habitats.

Ordinary Kriging is used on the temperature data in order to provide the temperature decadal layers. Kriging is an interpolation procedure using observations collected and a semivariogram to determine the values on non-sampled locations, and the procedures involved in kriging incorporate measures of error and uncertainty when determining estimations. Ordinary kriging is a type of kriging is a location-dependent weighted average of the observational valued from the given locations, where the weights depend upon the spatial correlation structure of the data [4]-[6].

Ordinary Kriging is a linear predictor [4], [5]:

$$
\hat{Z}\left(s_{0}\right)=\sum_{i=1}^{N} \lambda_{i} Z\left(s_{i}\right)
$$

where $s_{i}$ are locations with observation $Z\left(s_{i}\right)$ available and coefficients $\lambda_{i}$ satisfy the OK linear equation system,

$$
\left\{\begin{array}{c}
\sum_{j=1}^{N} \lambda_{j} \gamma\left(\varepsilon\left(s_{i}\right)-\varepsilon\left(s_{j}\right)\right)-\psi=-\gamma\left(\varepsilon\left(s_{i}\right)-\varepsilon\left(s_{0}\right)\right), i=1,2, \cdots, N \\
\sum_{j=1}^{N} \lambda_{j}=1
\end{array}\right.
$$

The Ordinary Kriging system is generated under the assumption of an additive spatial model,

$$
Z(s)=\mu(s)+\varepsilon(s)
$$

where $\mu(s)$ is the basic (expected) spatial trend and $\varepsilon(s)$ is a Gaussian error $N\left(0, \sigma^{2}(s)\right)$, i.e.,

$$
E[\varepsilon(s)]=0, V[\varepsilon(s)]=\sigma^{2}(s)
$$

Accordingly, the variogram $2 \gamma$ of the random error function $\varepsilon$ is just defined by:

$$
2 \gamma(h)=\mathrm{E}\left[(\varepsilon(s+h)-s(h))^{2}\right]
$$

where $h$ is the separate vector between two spatial point $s+h$ and $s$, [4], [5].

The decadal temperature layers reconstructed this way are named: 1960s temperature (1960-1969), 1970s temperature (1970-1979), 1980s temperature (1980-1989), 1990s temperature (1990-1999), and 2000s temperature (2000-2009).

The sample data of the common swift during each of the past five decades was reconstructed using published literature (in the form of bird club reports, checklists and journals), museum material and atlas data. Presence/absence data (per decade). The decadal swift data are reconstructed this way are: 1960s swifts (1960-1969), 1970s swifts (1970-1979), 1980s swifts (1980-1989), 1990s swifts (1990-1999), and 2000s swifts (2000-2009).

\section{PREDICTION AND RETRODICTION}

Species distribution models are used to estimate the relationship between species records at sample sites and the environmental and spatial characteristics of those sample sites [7]. The Species Distribution Model used in this study is MaxEnt [8]. MaxEnt applies Bayesian methods to estimate the potential geographic distribution of species by finding the probability distribution of maximum entropy and is an effective method for modelling species distributions from presence-only data [9].

The conventional Bayesian risk criterion is based on the quadratic loss function and use of a conjugate family, and the Maximum Entropy modelling is an important Bayesian inference, which is established by different risk criterion. MaxEnt is a Bayesian approach by which the species distribution probability is statistically estimated by searching the family of probability distributions under the maximum entropy criterion subject to environmental constraints.

The Gibbs family:

$$
q_{\lambda}(x)=\frac{1}{Z_{\lambda}(x)} \exp \left(\sum_{i=1}^{m} \lambda_{i} f_{i}(x)\right)
$$

with $\lambda_{i}$ being the weight parameters and $f_{i}(x)$ representing species $i$ 's probability distribution. Note that each element $x$ is a pixel of the investigated area. These probabilities $f_{i}(x)$ represent relative suitability of the environmental conditions in each pixel [8]-[10].

Using distribution and climate in the 1960s as the baseline, the MaxEnt Model were used to predict range changes of the swifts at decadal intervals, which were then compared with the observed ranges of the swifts at the same time intervals. In other words, current temperature and current species samples combined with future temperature, are used to predict future species distribution. And then the observed species distribution of the decade is produced to compare the predicted and observed species distributions. This technique 
is retrodiction rather than prediction, since we are using present data to explain past species distributions [11], [12].

The swift species sample data came from where the bird had been recorded during the previous decade; while the environmental variables that were used were the average temperature during the previous decade, and temperature for the decade in which the model attempted to predict distribution. The observed range of the swift in the 1960s was used as a climate envelope baseline. This allowed predictions of swift dispersion in subsequent decades which could be compared in a retrodictive fashion with observed distributions during each of those decades.

In Fig. 2, the predicted 1970s swift distribution is predicted using past 1960s swift samples, 1960s temperature layer, altitude layer and the future 1970s temperature layer.

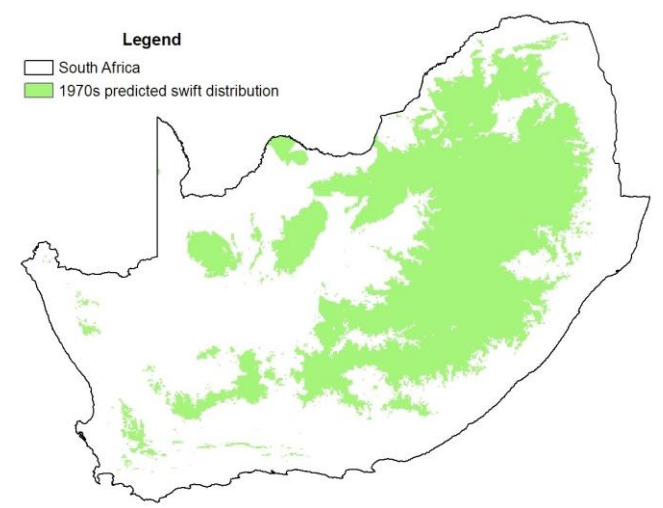

Fig. 2. Predicted swift range distribution using MaxEnt model for 1970s.

In Fig. 3, the actual or observed 1970-1979 swift distribution is predicted using the actual 1970s swift samples, altitude layer, and the current 1970s temperature layer.

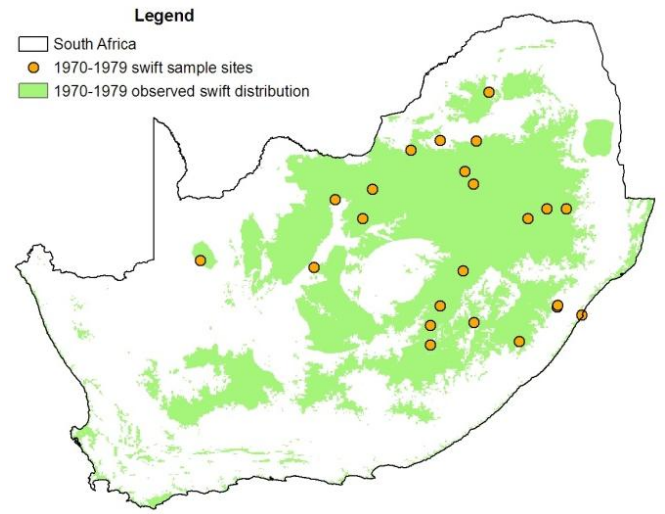

Fig. 3. Observed swift range distribution using bird samples for 1970-1979.

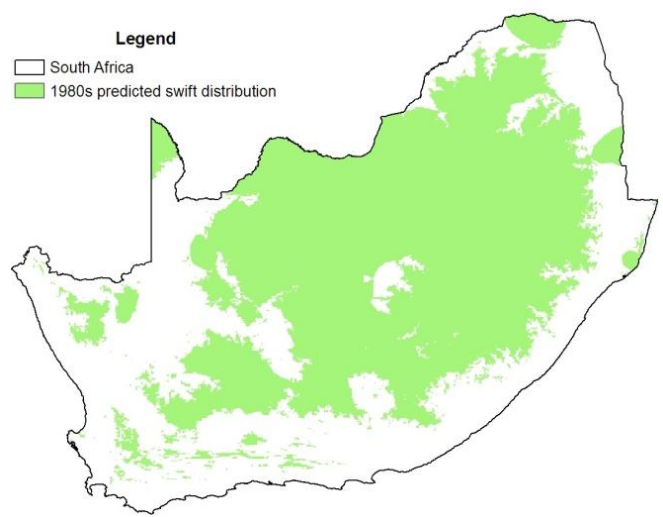

Fig. 4. Predicted swift range distribution using MaxEnt model for 1980s.
In Fig. 4, the predicted 1980s swift distribution is predicted using past 1970s swift samples, 1970s temperature layer, altitude layer, and the future 1980s temperature layer.

In Fig. 5, the observed 1980-1989 swift distribution is predicted using the actual 1980s swift samples, altitude layer, and the current 1980s temperature layer.

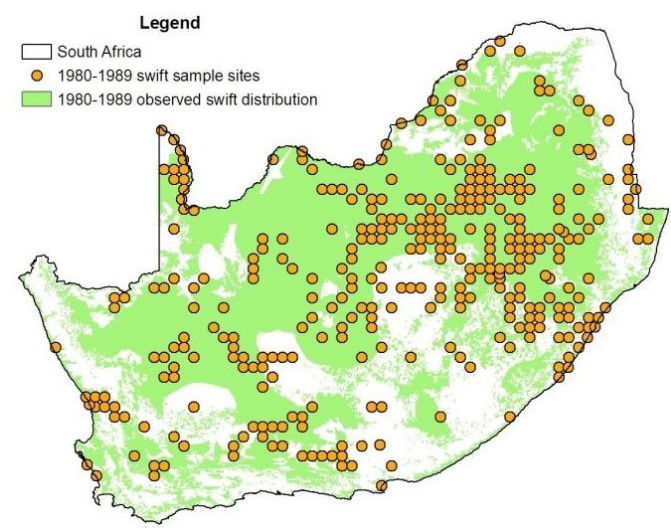

Fig. 5. Observed swift range distribution using bird samples for 1980-1989.

In Fig. 6, the predicted 1990s swift distribution is predicted using past 1980s swift samples, 1980s temperature layer, altitude layer, and the future 1990s temperature layer.

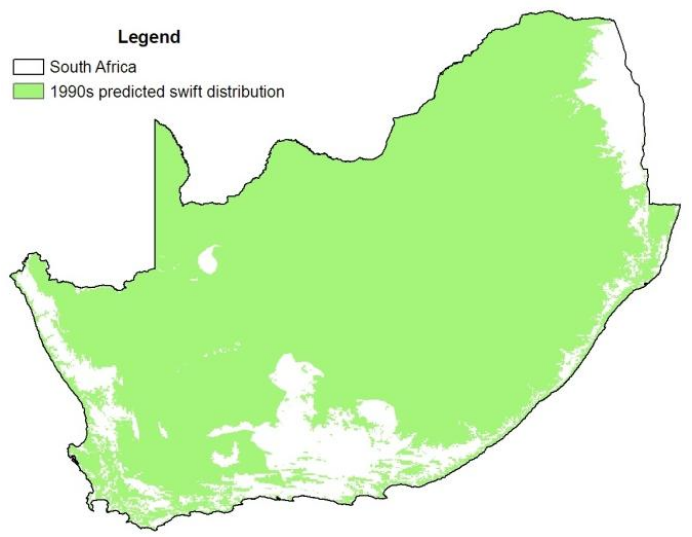

Fig. 6. Predicted swift range distribution using MaxEnt model for 1990s.

In Fig. 7, the observed 1990-1999 swift distribution is predicted using the actual 1990s swift samples, altitude layer, and the current 1990s temperature layer.

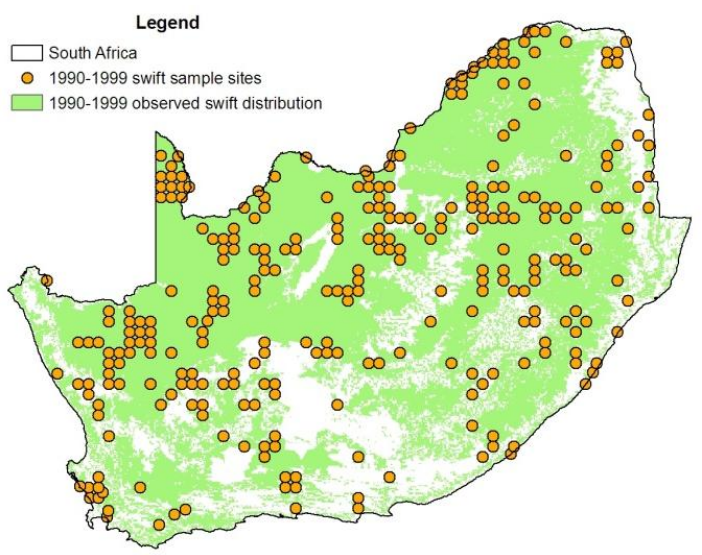

Fig. 7. Observed swift range distribution using bird samples for 1990-1999.

In Fig. 8, the predicted 2000s swift distribution is predicted using past 1990s swift samples, 1990s temperature layer, altitude layer, and the future 2000s temperature layer. 


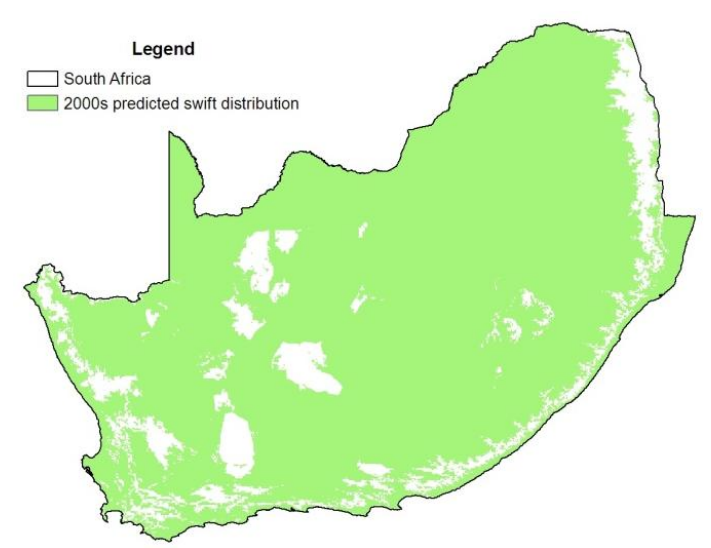

Fig. 8. Predicted swift range distribution using MaxEnt model for 2000s.

In Fig. 9, the observed 2000-2009 swift distribution is predicted using the actual 2000s swift samples, altitude layer, and the current 2000s temperature layer.

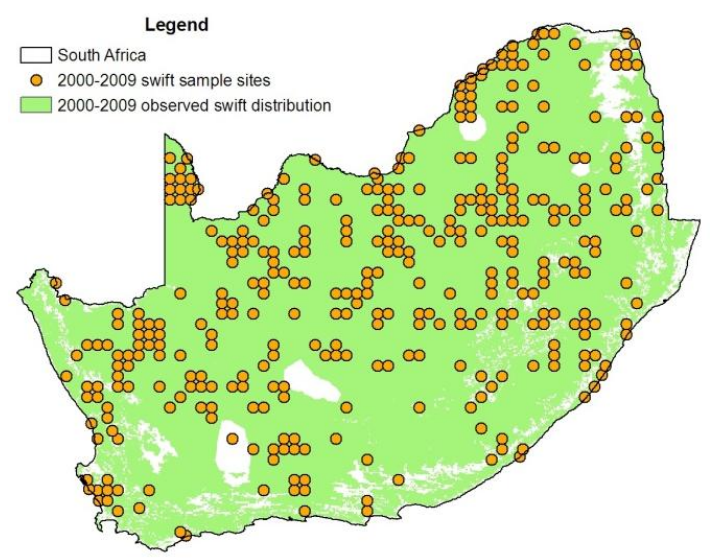

Fig. 9. Observed swift range distribution using bird samples for 2000-2009.

In the 1970s (Fig. 2 and Fig. 3), based on the climate of the 1960s, swifts were expected to occur over a larger area of South Africa than was the case. This apparent mismatch between swift distribution and climate suitability could have several explanations. This could be the patterns of swift distribution from which they are derived are based on rather few empirical records, especially in the 1960s, which formed the temporal baseline for this study. And not all weather stations included in the analysis for the 1960s had full decadal records. Therefore the potential climate envelopes in the 1970s seemingly available to the swifts might be more generous than reality.

By the 1980s (Fig. 4 and Fig. 5), the distributional database for swifts was more comprehensive and there was better coverage of the country by weather stations. This was the decade in which swifts underwent the greatest range expansion, with major movement into the west of the country. The predicted distribution is well matched, but we notice the actual observed expansion is greater than the predictions, which indicates a quick response of the swifts to temperature changes. That the model predicted the westward range expansion so well implies that this major expansion was triggered by a major climatic change.

In the 1990s (Fig. 6 and Fig. 7), as in the previous two decades, the range of the swifts was again predicted to expand, extending well into the Western Cape, reaching as far as the Cape Peninsula. Areas predicted to have a high probability of swift occurrence also expanded in a south-westerly direction. With a few outliers, swift distribution in the 1990s largely conformed to model predictions. The expected range of swifts in the 1990s was fairly similar to the predicted range, the main difference being a major expansion of the climate window where a high probability of occurrence was expected as far as the south-west coast. In terms of the birds' observed distribution, there was little change from the 1980s, the only noteworthy exception being the first appearance of swifts along the south coast. The majority of swift records fell within their expected climate envelope, and were clustered in areas of high suitability. In the 1990s, swifts were expected to avoid areas below the $17.5^{\circ} \mathrm{C}$, favouring temperatures in the range $17.5-24.5^{\circ} \mathrm{C}$.

In the 2000s (Fig. 8 and Fig. 9), with few exceptions, and based on climate data alone, swifts were predicted to occur throughout South Africa by the 2000s. The observed dispersion of swifts largely followed this prediction, but with an almost complete avoidance of the few remaining areas predicted to be unsuitable. The expected climate envelope of occupancy and predicted range in the 2000s were almost identical. The full extent of the climatically suitable area was exploited by the birds, and they continued to avoid areas outside this envelope. Unsurprisingly, the swifts are predicted to occur between the $17.3^{\circ} \mathrm{C}$ and $23^{\circ} \mathrm{C}$ isotherms.

In the all the predicted species distribution, they match fairly closely with the reality of actual species distribution. The overall rising warm temperatures over the past several decades due to climate change, causes the rapid expansion of swifts moving into regions which are previously too cool for the species. This provides evidence for a major range change of a bird species in South Africa being driven primarily by climatic factors [11], [12].

\section{DISCUSSION}

The range change between the 1970s and 1980s was by far the most significant of the past 50 years. In the space of less than ten years, swifts had penetrated previously uncolonised south-western regions of the country. This rapid range expansion, approximately a doubling of the species' regional range in less than a decade, is unusual for birds. However, range changes by most terrestrial birds are constrained by a far broader suite of habitat factors (nest sites, roost sites), but the common swifts (constrained solely by food) are entirely aerial and are non-breeding seasonal visitors. Although the predominant direction of range expansion by the swifts has been westward, this is because they are opportunistically occupying areas that are becoming suitable by virtue of rising temperatures.

The fact that the swifts' historical range was confined to the summer-rainfall area and its present range spans both summer-rainfall and winter-rainfall areas, strongly supports the conclusion that the species' historical distribution was determined by factors other than rainfall seasonality. Its range expansion support the prediction [13] that new species' ranges are expected to centre around a core area that is most similar to their climate envelopes prior to climate change.

Following the rapid climate change in South Africa in the 1970s and 1980s, the situation appears to have become more 
stable, with swifts having similar predicted ranges and patterns of occurrence in both the 1990s and 2000s. There has been an overall cooling of the west of the country in the 2000s, while temperatures in the east have continued to rise.

The range expansion of the common swift also accords to the prediction [14] that, if limited by temperature, populations should respond to climate warming by expanding into nearby habitats that were previously too cool. Our findings strongly support the hypothesis that climate change has indeed been the primary driver behind the range expansion of common swifts. The most probable primary link between swifts and climate is the species' aerial, invertebrate prey and their dependence on temperature. Under scenarios of climate warming, insects are expected to pass through their larval stages faster and metamorphose to adulthood earlier [15]. If this is the case, then aerial insects could also be expected to have tracked the warmer band of temperature extending southward and westward across the country. However, there is no data against which to test this hypothesis.

The combination of prediction and retrodiction used in this study provides some compelling evidence in support of common swifts having changed their South African distribution in response to the changing thermal landscape of the region. That the sequential ranges predicted using a modelling approach were very close to those occupied by the swifts means that the model does perform the task of predicting future swift habitat ranges, as verified by retrodiction. An important advantage of this study is that the future distribution range is known, making the evaluation more objective.

Whenever a predictive or retrodictive modelling approach is used, the accuracy of the distributional database can be a potential source of error [8]. Such error could exist in this case, especially with the early swift data, because this species can readily be confused with African black swift [16]. The database from the 1960s is likely littered with false negatives, as may be that from the 1970s. There are also limitations attached to the climatic data, especially sparsely recorded temperature records from 1960s and 1970s. Despite these limitations, which apply to many similar studies, the species distribution model provides compelling evidence that the swift population in South Africa are driven by temperature changes.

The most important conclusion to be drawn from this study is that the swift species reacted sufficiently quickly to climate change that is satisfies the requirements of a indicator species. The reason it does so and the reason for its selection, is that its behaviour on the non-breeding grounds is influenced primarily by a single variable-the availability of small aerial insects. Coupled with this, it is highly mobile, being both a long-distance migrant and, on the non-breeding grounds, a nomadic opportunist [8].

Analyses of recent range shift changes by South African birds have been unable to link these changes in a convincing way to climate change. Previously research studies champion human-mediated, landscape-level change as the primary driver of recent avian range changes. This study thus provides the first compelling evidence for a major range change of a bird species in South Africa being precipitated primarily by climatic factors. The predicted results show that the species distribution modelling approach can predict the future patterns of range change of a species, given a good data set.

\section{CONCLUSION}

The range expansion of the common swift support the prediction of that, if temperature is the primary explanatory variable, populations will respond to climate warming by expanding into nearby habitats that were previously unsuitable due to lower temperatures. Our findings strongly support the hypothesis that climate change has indeed been the primary driver behind the range expansion of Common Swifts. However, what is less certain is the proximate factor underlying the response to the change in climate. The conclusion drawn from this study is that the bird species reacted very quickly to climate change, and this could be due to availability of food, namely small aerial insects.

The primary aim of the study was to generate parallel reconstructions over time of the changing range of common swift and the changing climate of South Africa, and to assess whether a species distribution modelling approach could have predicted the future patterns of range change. The parallel reconstructions have been produced, and using the predictive and retrodictive techniques, it has been proven that the species distribution model can indeed predict the future swift distributional range change. This study also represented the first empirical test of whether a South African bird's range has been influenced by climate change.

\section{ACKNOWLEDGMENT}

We thank Mzukisi Gwata from the South African Weather Service (SAWS), and Chris Lennard and Lisa Coop from CSAG for providing the historical temperature data used in this study.

\section{REFERENCES}

[1] PhpBB Group. (March 2014). Africa Wild Bird Book - The Second Edition. [Online]. Available: http://www.sagr.co.za/forum/viewtopic.php?p=183756

[2] W. J. M. Hagemeier and M. J. Blair, The EBCC Atlas of European Breeding Birds, London, T \& AD Poyser, 1997.

[3] A. A. Hoffman and P. A. Parsons, Extreme Environmental Change and Evolution, Cambridge: Cambridge University Press, 1997.

[4] D. Guo, R. Guo, and C. Thiart, "Predicting air pollution using fuzzy membership grade kriging," Journal of Computers, Environment and Urban Systems, Elsevier, vol. 31, no. 1, pp. 33-51, 2007.

[5] D. Guo, R. Guo, C. Thiart, and T. Oyana, "GM(1,1)-kriging prediction of soil dioxin pattern," Representing, Modeling and Visualizing the Natural Environment: Innovations in GIS 13, Taylor \& Francis Group, Florida: CRC Press, 2009, pp. 243-253.

[6] D. Guo, R. Guo, C. Thiart, and Y. H. Cui, "Imprecise uncertainty modelling of air pollutant PM10," Advanced Air Pollution, InTech, 2011, pp. 193-212

[7] J. Franklin, Mapping Species Distributions: Spatial Inference and Prediction, Cambridge, UK: Cambridge University Press, 2009.

[8] S. J. Phillips, R. P. Anderson, and R. E. Schapire, "Maximum entropy modeling of species geographic distributions," Ecological Modelling, vol. 190, pp. 231-259, 2006.

[9] J. Elith, S. J. Phillips, T. Hastie, M. Dudík, Y. E. Chee, and C. J. Yates, "A statistical explanation of MaxEnt for ecologists," Diversity and Distributions, vol. 17, pp. 43-57, 2011.

[10] S. J. Phillips, M. Dudík, and R. E. Schapire, "A maximum entropy approach to species distribution modeling," in Proc. the 21st International Conference on Machine Learning, pp. 655-662, 2004.

[11] M. Kuhlmann, D. Guo, R. Veldtman, and J. Donaldson, "Consequences of warming up a hotspot: Species range shifts within a 
centre of bee diversity," Diversity and Distributions, Blackwell Publishing Ltd, vol. 18, issue 9, pp. 885-897, September 2012.

[12] M. McLeish, D. Guo, S. van Noort, and G. F. Midgley, "Life on the edge: rare and restricted episodes of a pan-tropical mutualism adapting to drier climates," New Phytologist, vol. 191, issue 1, pp. 210-222, July 2011.

[13] B. F. N. Erasmus, A. S. L. Jaarsveld, S. L. Chown, M. Kshatriya, and K. J. Wessels, "Vulnerability of South African animal taxa to climate change," Global Change Biol., vol. 8, pp. 679-693, 2002.

[14] C. D. Thomas, S. E. Williams, and A. Cameron, "Extinction risk from climate change," Nature, vol. 427, pp. 145-48, 2004.

[15] J. Peñuelas and I. Filella, "Responses to a warming world," Science, vol. 294, pp. 793-795, 2001.

[16] P. A. R. Hockey, W. R. J. Dean, and P. G. Ryan, Roberts - Birds of Southern Africa, 7th ed., Cape Town, Trustees of the John Voelcker Bird Book Fund, 2005

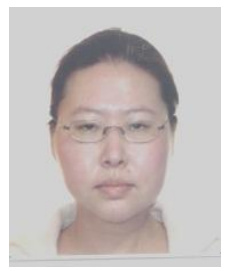

Danni Guo is a senior scientist at the Biodiversity Research Assessment and Monitoring, South African National Biodiversity Institute, Kirstenbosch Research Center, Private Bag X7, Claremont 7735, South Africa. She has received the master of science from University of Durham (United Kingdom) in 2003, and the Ph.D. in statistical sciences from University of Cape Town (South Africa) in 2007. Since 2007 she held the position of Spatial Modeller as a senior scientist, within the Statistical Ecology Division, Biodiversity Research Assessment and Monitoring, South African National Biodiversity Institute.

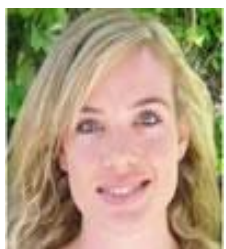

Gina Zietsman was a master student with the Percy FitzPatrick Institute, DST/NRF Centre of Excellence, University of Cape Town, Rondebosch 7701, South Africa. She is now working for the South African company-the Moss Group, specialising in integrating sustainability into business strategies.

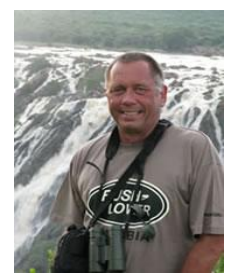

Philip A. R. Hockey was the director of the Percy FitzPatrick Institute, DST/NRF Centre of Excellence, University of Cape Town, Rondebosch 7701, South Africa. He moved from England to South Africa in 1979 to study African Black Oystercatchers for his $\mathrm{PhD}$. After graduating in 1983, stayed on at the FitzPatrick Institute as a lecturer. He was appointed the institute's director in 2008 and has led the DST/NRF Centre of Excellence to new heights. His contribution in this regard was recognised by the South African Network for Coastal and Oceanic Research who named him 'Marine and Coastal Communicator of the Year' in 2000 and he was awarded the Stevenson-Hamilton Medal by the Zoological Society of Southern Africa in 2008. 